\title{
Towards a Gamified Recommender System for the Elderly
}

\author{
Madita Herpich \\ Hochschule Augsburg \\ Augsburg, Germany \\ madita.herpich@informatik.uni-augsburg.de \\ Andreas Seiderer \\ Universität Augsburg \\ Augsburg, Germany \\ andreas.seiderer@informatik.uni-augsburg.de
}

\author{
Thomas Rist \\ Hochschule Augsburg \\ Augsburg, Germany \\ thomas.rist@hs-augsburg.de \\ Elisabeth André \\ Universität Augsburg \\ Augsburg, Germany \\ andre@informatik.uni-augsburg.de
}

\begin{abstract}
Starting from our previous work on a digital picture frame - the CARE system - that interleaves a picture display mode with a recommender mode to promote a healthy life-style and to increase well-being of elderly people, this paper investigates the use of gamification as a means to increase user appreciation of the CARE system. To this end, we arranged two co-design workshops with peer-groups of senior citizens. We report on outcomes of the workshops and draw conclusions for a gamified version of CARE.
\end{abstract}

\section{CCS CONCEPTS}

\section{- Human-centered computing $\rightarrow$ Human computer interac-} tion;

\section{KEYWORDS}

technologies for health and well-being

\section{ACM Reference format:}

Madita Herpich, Thomas Rist, Andreas Seiderer, and Elisabeth André . 2017. Towards a Gamified Recommender System for the Elderly. In Proceedings of DH'17, fuly 2-5, 2017, London, United Kingdom, , 5 pages.

DOI: http://dx.doi.org/10.1145/3079452.3079500

\section{INTRODUCTION}

In the project CARE (Context-Aware Recommender System for the Elderly [10] [4]) we augmented a digital picture frame with a recommender mode. In picture frame mode, the CARE system retrieves pictures (including various motives, such as landscapes, flowers, pets, etc.) and photographs of family members for display. When switched to recommender mode, CARE provides hints on activities that potentially contribute to increase the user's wellbeing. To this end, CARE draws on a dimensional well-being model and keeps a repository of prefabricated recommendations for each well-being dimension (cf. left-hand side of Fig. 2). For instance, CARE may give hints on how to increase comfort and ease of the living environment, or suggest engagement in physical or mental activities to increase physical and mental well-being. Fig. 1 shows

(c) Author | ACM 2017. This is the author's version of the work. It is posted here for your personal use. Not for redistribution. The definitive Version of Record can be found at DOI: http://dx.doi.org/10.1145/3079452.3079500 a CARE frame mounted on a shelf and a user following instructions for a physical excercise.

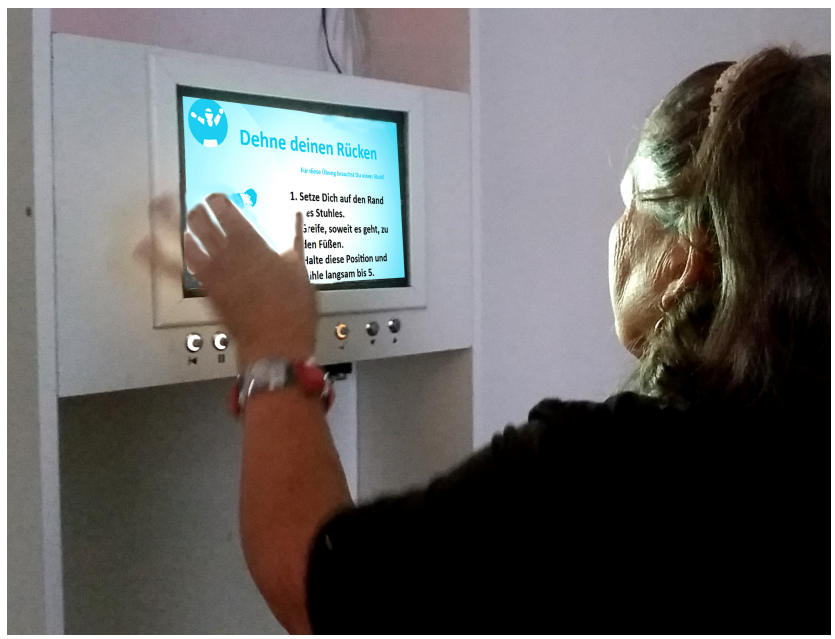

Figure 1: CARE digital image frame mounted on a shelf

With the ambition to increase the effectiveness of CARE, it was questioned, in what way well-known computer game elements and principles, such as live feedback and especially rewards, may be used to enhance the user's overall appreciation of the CARE system. Our initial approach towards a gamified version of CARE is sketched in Fig. 2. Basically the idea is to link recommendations given by CARE with some measurable indicators of whether or not recommendations have been followed. For instance, if CARE suggests a physical exercise, possible indicators are the number of repetitions or just elapsed exercising time.

Our working hypothesis was that a uniform rewarding scheme across all kind of recommended activities would increase the users' perception of CARE as a coherent recommendation system. Therefore, measured indicators for different recommendations would be mapped onto abstract points, which in turn would be mapped to a rewarding scheme of choice, such as score boards, badges or virtual currencies. Scores encompass rewarding in form of comparison with one self, like a high-score in speed races, and with other players in a leaderboard. Badges are a means to represent the status of each player (CARE user) by providing him or her with awards or the recognition by others. The virtual currency category 


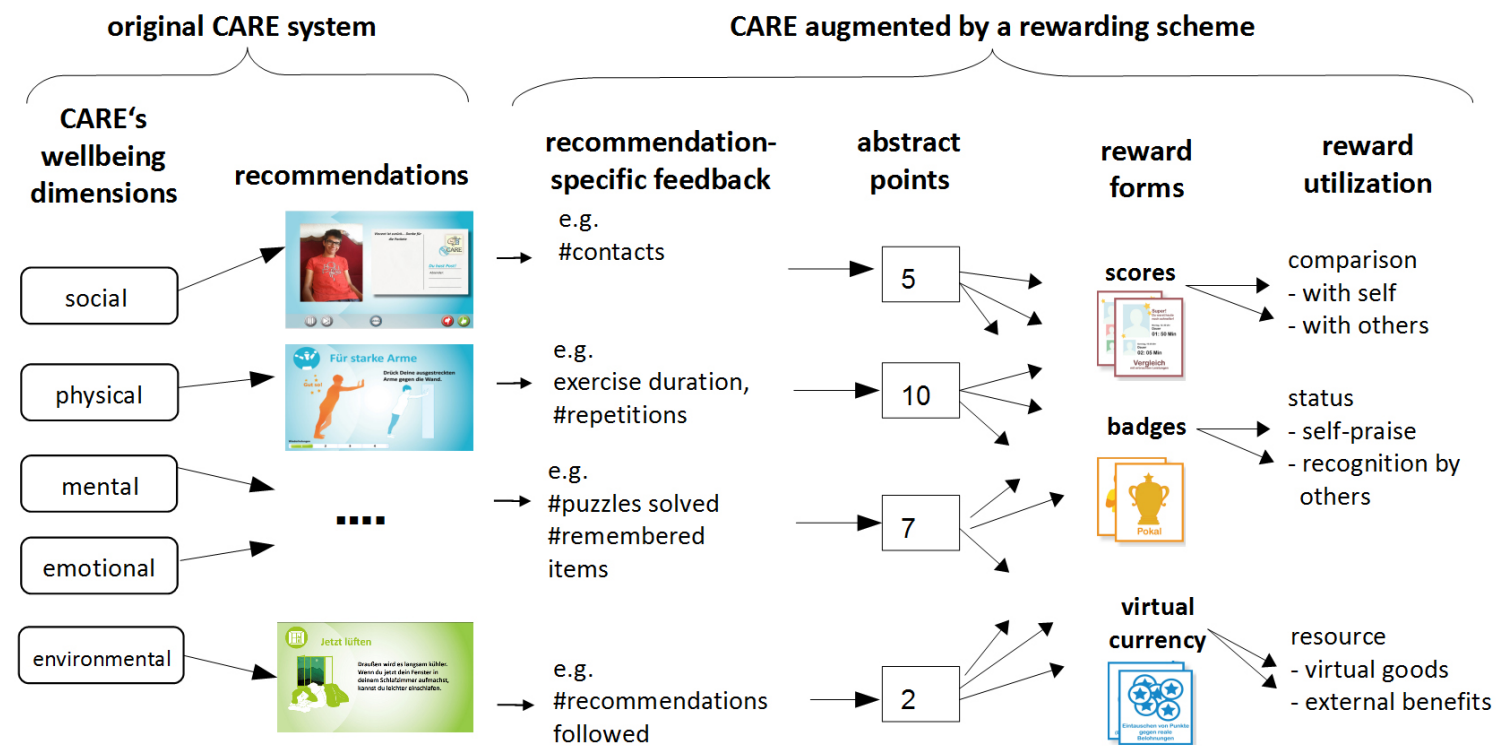

Figure 2: Initial proposal for a gamified CARE system

stands for all kind of rewards which can be exchanged with virtual or real goods. For instance, virtual coins may be used to purchase yet needed pieces of a jigsaw puzzle on screen or to have a virtual plant grow and prosper. Another option would be to use the coins for purchasing real-world goods, such as a cup of coffee.

However, there was uncertainty which activities recommended by CARE should get rewarded at all, and what kind of rewards users would find appropriate. To shed light on these questions we arranged two co-design workshops with seniors, method and outcomes will be reported in Sec. 3 .

\section{RELATED WORK}

Following the definition by Deterding et al. [2], gamification is referred to as ".. the use of game design elements in non-game contexts". A prominent class of such game design elements are all kinds of rewarding scheme. Based on work by Hallford and Hallford [3], Wang et al. [12] distinguish between eight different forms of rewards: "Score systems", "Experience point reward systems", "(Virtual) Item granting systems", "(Virtual) Resources", "Achievement systems" referred to "titles", "Feedback messages", "Plot animations and pictures", "Unlocking mechanisms" or "Access".

Of high relevance for our work on the CARE system are studies and projects that address gamification to promote a healthier lifestyle and to increase overall well-being. This includes various gamified applications for physical and mental training - sometimes called exergames or active video games - as they can be found in the e-Health sector. Klompstra and colleagues [6] reviewed 11 studies focusing on the influence of exergames on older adults' physical activity. They concluded that exergames increase physical activity in elderly individuals, stroke patients and cardiac patients, but felt that further testing is needed, e.g. to examine long-term effects of exergame platforms. Lumsden et al. [7] reviewed 33 studies on gamified applications for cognitive training and assessment purposes.
They identified a number of different reasons for deploying game elements in cognitive training and testing, including the aim to increase the users' motivation and long-term engagement, as well as usability, intuitiveness and appeal of an application. In a more recent literature research Johnson et al. [5] revisited 19 papers that report empirical evidence on the effect of gamification on health and well-being including both physical as well as mental exercises. While the majority of studies reported positive effects, about 41 per cent reported mixed or neutral effects especially with regard to cognitive outcomes.

Meta studies as those referenced above provide a good link to cases of successful and less successful gamification approaches. However, they do not explain why gamification worked or failed. We refer to Zuckerman et al. [13] as an example of a study that did not show a positive effect of gamification on users' motivation to engage in physical exercising. As a potential reason, they indicated that most users did not understand the points' allocation mechanism, but considered them as meaningless. In order to better understand how game design elements motivate user behavior in non-game contexts, several attempts have been made to discuss the role of incentives and rewards as found in computer games based on psychological theory. Such an approach is presented by Richter et al. [9]. Important to their analysis is the Self-Determination Theory [11] that distinguishes between intrinsically motivated behaviours, which are performed out of interest and satisfy the innate psychological needs for competence and autonomy, and extrinsically motivated behaviours, which support activities (e.g., collecting points) that are done in order to attain some outcome separable from the primary task (e.g., physical exercising). Richter et al. consider the use of points, badges, leaderborads etc. as applications of extrinsic motivators. They conclude that careful selection and implementation of extrinsic motivators will trigger internal motivation and aid in maintaining it. However, they point out that 
success in one non-game context does not guarantee that the same mechanism will be successful in another non-game context.

While the majority of studies suggest that gamification works in many cases, it still remains a challenge for application designers to decide on which gamifying elements should be added because they are likely to add value to an application, and which one should be neglected since they may even cause opposite effects. Suggestions for application design can be extracted from works compiling gamification guidelines based on interviews with seniors [1].

\section{CO-DESIGNING CARE WITH SENIORS}

\subsection{Objectives}

To obtain stimulating input on how to gamify the existing CARE system we arranged two workshops (W1, W2) with elderly people, having in mind the following objectives:

(1) to learn about the daily routines of the participants and whether they already engage in activities that promote a healthy lifestyle,

(2) to elicit feedback on technology that actively gives recommendations and suggests activities,

(3) to gather opinions on what could establish a meaningful rewarding scheme for recommended activities,

(4) to investigate contextual factors, such as different living environments (at home vs. in retirement home).

\subsection{Participants and Working Materials}

Participants: For a first workshop (W1) we established contact with a local seniors' association and recruited a group of 11 women and one man of different age between 60 and 70. All were retired, live independently and maintain their own households. In contrast, for the second workshop (W2) we recruited nine residents (8 women, one man) of a retirement home. All members of this group were in the $80+$ age group. Compared to W1 participants, members of this age group were notably suffering more strongly from age-related impairments, such as limited mobility and lower levels of cognitive performance.

Worksheet and Cards. To elicit information about the participants' daily routines and activities, and to reveal their attitudes towards rewarding schemes, a working sheet with a timeline and three piles of cards has been prepared. The first pile consisted of cards depicting daily routines. Cards of the second pile showed activities that CARE would recommend to promote a healthier lifestyle, e.g., physical or mental exercises. By means of cards from the third pile, participants could allocate different kinds of reward to activities. While there is a huge variety of possible rewards, we focused on three types of reward, which we found suitable for CARE: scores, badges and virtual currencies (cf. right-hand column of Fig. 2). Finally, a number of blank cards were included so that participants could use them to write down additional activities or rewards for which no cards had been prepared. With exception of blank cards, the visual appearance of all cards resembled the graphical style used for screen content displayed by the CARE system.

Predefined questions for semi-structured interviews. Semi-structured interviews were prepared in which participants would be involved at several stages of the workshop to receive feedback on a number of questions, such as whether participants already care about a healthy lifestyle and how this is mirrored in their daily routines, what kind of reward schemes participants are already familiar with, or what they think about a recommendation system like CARE.

CARE-instructed physical exercises. To enable "hands-on" familiarization with the CARE system, participants were given the opportunity to engage in two physical exercises, inspired by Panton and Loney's instruction book for health care providers [8]. The first one was a seated arm raise exercise using lightweight dumbbells to strengthen triceps muscles. CARE instructs the exercise through keyframe animations showing arm movements to be performed, see Fig. 3. To monitor progress and accuracy of practice, a user wears a Microsoft Band 2 for recording accelerometer data. The second exercise was for training balance. The user steps on a pressure-sensitive floor mat, slowly raises one heel off the ground, holds for a second, and then returns to starting position for another round with the other heel. The exercise is textually instructed while key-frame animations mirror the actual execution of the exercise by the practitioner.

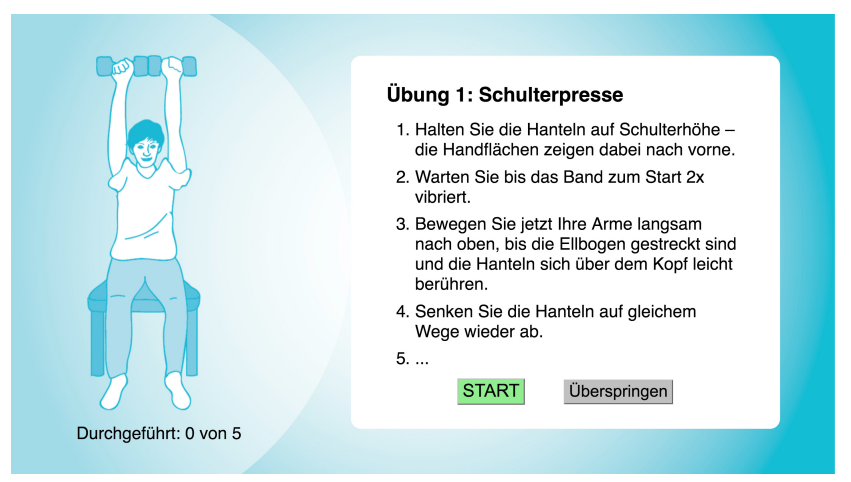

Figure 3: Screens of the practice with dumbbells

\subsection{Procedure}

Workshop W1 took place at a senior meeting centre, while W2 was held in an assembly room of the retirement home. Both workshops were administered by one female and two male experimenters. In the workshops, participants:

- received an introduction to the CARE project and the CARE prototype including the opportunity to practise instructed and monitored by the CARE prototype,

- got an explanation of their role in helping us extend and improve the CARE system,

- took part in three subsequent card-laying tasks to give us an idea about their daily routines and activities, and to reveal their attitudes towards rewarding schemes,

- were asked to provide verbal feedback on a number of predefined questions which are to be prompted by the experimenters during the workshop.

Throughout the workshop the experimenters performed data gathering by taking notes on verbal responses and comments, and by 
taking pictures of the outcomes of the card-laying tasks. Grouping and analysis of the recorded material took place after the workshop.

\subsection{Outcomes and Observations}

Task 1: Daily routines. All participants described their daily lives by arranging activity cards on a timeline, starting with getting up in the morning and finishing with going to sleep in the evening. All participants structured their daily routines into morning (prelunch) activities and after-lunch activities. None of the participants expressed a need to use a blank card for routine activities not yet depicted on pre-prepared cards. A comparison of the resulting worksheets among participants within the same workshop and between workshops revealed moderate variations in both number and kind of mentioned activities from one participant to the other. Participants of W1 indicated cleaning and shopping being part of their agendas, but typically before lunch (for cleaning in 12/12 and, for shopping in 11/12 cases). In view of CARE we were in particular interested in activities that potentially contribute to a healthier lifestyle and increased well-being. To this end, we counted the number of such activities on the agendas. Regarding before-lunch activities of W1 participants, only doing sports (7/12) fits into this category, while after lunch all W1 participants indicated at least 2, and in one case even 5 of such activities (including taking a nap, going out for a stroll, visiting friends, doing sports).

In contrast, as there is no need for W2 participants to maintain an own household, activities such as cleaning (0/9), cooking (0/9), and shopping (3/9) were not or only rarely part of daily routines. Rather, W2 participants incorporated more leisure-related activities into their working sheets, for example going out for a stroll in the morning (5/9) or even watching TV in the morning (3/9). Some participants (4/8) included the "hobby" card into their agendas to refer to hobby-related activities that were not covered by prepared cards. In these cases reference was made to activities, such as joint singing, card playing, or knitting which are regularly arranged by staff members of the retirement home in the afternoon.

Task2: Appreciated recommendations. In response to Task2, all participants augmented their agendas by recommendations for activities as they could be provided by CARE. Among W1 participants we observed great variations in number (between 2 and 8 cards, average 4) and kind of wanted recommendations. In the morning, recommendations for airing the room (9/12) and for doing sports $(7 / 12)$ were most frequently mentioned, while for the second half of the day recommendations for relaxing scored highest (9/12). To our surprise, none of the participants wanted to receive recommendations related to social activities, such as visiting friends. We also examined whether selected recommendations were related to activities already included in a participant's agenda (cf. Task1) to see whether a recommendation either serves as a reminder to do something that one usually does, or as a stimulation to do something in addition to daily routines. In case of recommending physical exercising those who already did sports appreciated reminders to carry on, and they were also open to receive stimulation for further physical exercises. Recommendations of relaxation-related activities were classified as "stimulators" as none of such activities were included in any agenda after competition of Task1.
W2 participants enriched their agendas with between 2 and 8 recommendations. Quite similar to W1 participants, recommendations for airing the room (7/9) and for doing sports (4/9) in the morning were most frequently mentioned. Chosen recommendations for afternoon activities included almost all available categories, but varied greatly among participants. Three participants included cards that recommend activities with friends or other residents in contrast to W1 particpants who did not choose such cards at all. When interviewed the three participants said that they would appreciate reminders on scheduled afternoon activities, such as joining the singing or card playing group.

Task3: Rewards for activities. Regarding reward schemes that participants already knew, some referred to their times in school where they earned sport badges, stickers or achievement stamps. Most were also familiar with commercial rewarding schemes, such as Pay Back cards handed out by some supermarket chains. Further, known types of rewards were praise by friends for having cooked a good meal for them, or just receiving a phone call after a nice visit of a friend or family member. One W1 participant felt rewarded when her grandchildren got good marks in school because she helped them with doing their homework. Also, there was common agreement among the participants of both workshops that it is often the activity itself or its outcome that constitutes a tangible reward to them. Examining W1 and W2 worksheets to see which activities got associated with rewards (scores, badges, or virtual currency) revealed a quite heterogeneous picture. Only 4 of $9 \mathrm{~W} 2$ participants wanted to receive rewards at all. Those who appreciated receiving rewards expressed a preference for virtual currency to earn tangible rewards, while two of them were also open to receive badges.

W1 participants were more open towards the idea of receiving rewards. Only one W1 participant did not assign any reward arguing that having accomplished an activity is enough rewarding itself and doesn't need an extra reward on top of it. Three W1 participants rewarded only activities which were already part of their agendas (such as cleaning, gardening, or surfing the Internet as this was considered a challenging activity), but not to recommended activities (cf. Task 2). In contrast, seven W1 participants only rewarded recommended activities, and only one participant rewarded both, routine activities as well as recommended activities (cf. lefthand worksheet shown in Fig. 4).

With regard to reward types we observed among W1 participants a strong preference (21 cases) for virtual currencies that could be used to purchase real or virtual goods. Several suggestions were given for potential exchanges, such as using collected virtual currency for sports equipment. Badges were assigned in 6 cases, and scores in another 5 cases. However, only three W1 participants rewarded activities consistently with the same type of reward, while all others suggested different reward types for different activities. Also, we did not observe dominant occurrences of activity/reward associations among all $\mathrm{W} 1$ and $\mathrm{W} 2$ participants, but a variety of individual preferences.

Feedback on exercises. In total, 7 women and one men participated in both exercises. In the balance exercise, participants stepped on the floor mat following textual instructions on screen. In addition, participant's movements were mirrored by an animated 

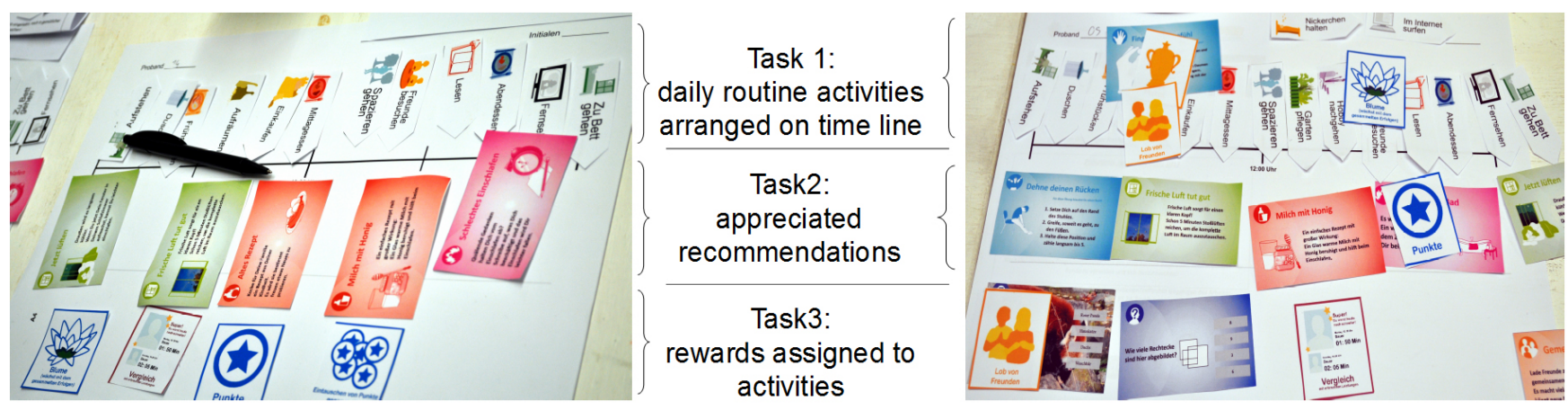

Figure 4: Two of the finished worksheets

character. However, some participants found this kind of feedback more confusing than helpful.

For the dumbbells exercise participants wore a bracelet with accelerometer sensors and were guided through three different exercises, which all started with a short vibration of the wearable. In the case of the arm raise exercise all participants appreciated the animated instructions as they could synchronize with the depicted movements. Wearing the Microsoft Band 2 bracelet was rated unintrusive, however, putting it on tightly attached required assistance by the experimenter. After finishing each exercise participants were rewarded with points, depending of her/his movements and the percentage of exercise completion. In the current implementation, a score simply reflects the percentage at which an excercise was completed as instructed. For example, a participant would receive 60 points out of 100 if 10 repetitions were instructed, but only 6 were actually performed. However, this rewarding scheme was not understood by all participants.

\section{CONCLUSIONS}

Albeit the fact that our two peer groups suffered from gender unbalance, we consider the feedback gathered from workshop participants a valuable source to drive the further development of our CARE system. First of all, we feel encouraged to incorporate a reward mechanism into CARE. However, we no longer think that a uniform rewarding scheme across all recommendation types is the approach of choice. Therefore, we will give users the opportunity to configure the rewarding schemes individually. In configuration mode, users should be enabled to easily select activities and associate them with reward types that they find suitable.

With regard to physical exercises, we plan to run a further codesign workshop to explore options for meaningful mappings of performance measures to rewards. In particular, degrading performance, for example, due to age or illness, should be considered in the rewarding mechanism to avoid user frustration.

\section{ACKNOWLEDGMENTS}

We thank the members of the "Mehrgenerationstreffen" of the ASB Göggingen who participated in workshop W1, and the residents of Anna Hintermayr-Stift Augsburg taking part in workshop W2. This research was partly funded by the Bavarian State Ministry for
Education, Science and the Arts (STMWFK) as part of the ForGenderCare research association and by the German Federal Ministry of Education and Research (BMBF) under grant number 01XZ13022.

\section{REFERENCES}

[1] Bob De Schutter and Vero Vanden Abeele. 2010. Designing Meaningful Play Within the Psycho-social Context of Older Adults. In Fun and Games '10. ACM, New York, NY, USA, 84-93.

[2] Sebastian Deterding, Dan Dixon, Rilla Khaled, and Lennart Nacke. 2011. From Game Design Elements to Gamefulness: Defining "Gamification". In MindTrek '11. ACM, New York, NY, USA, 9-15.

[3] Neal Hallford, Jana Hallford, and Andre LaMothe. 2001. Swords and Circuitry: A Designer's Guide to Computer Role-Playing Games. Premier Press, Incorporated.

[4] Stephan Hammer, Andreas Seiderer, Elisabeth André, Thomas Rist, Sofia Kastrinaki, Charline Hondrou, Amaryllis Raouzaiou, Kostas Karpouzis, and Stefanos Kollias. 2015. Design of a Lifestyle Recommender System for the Elderly: Requirement Gatherings in Germany and Greece. In PETRA '15. ACM, New York, NY, USA, Article 80,8 pages.

[5] Daniel Johnson, Sebastian Deterding, Kerri-Ann Kuhn, Aleksandra Staneva, Stoyan Stoyanov, and Leanne Hides. 2016. Gamification for health and wellbeing: A systematic review of the literature. Internet Interventions 6 (2016), 89-106.

[6] Verheijden Klompstra, Leonie, Jaarsma, Tiny, and Anna Strömberg. 2014. Exergaming in older adults: A scoping review and implementation potential for patients with heart failure. European fournal of Cardiovascular Nursing 13, 5 (2014), 388-398.

[7] Jim Lumsden, Elizabeth A. Edwards, Natalia S. Lawrence, David Coyle, and Marcus R. Munafò. 2016. Gamification of cognitive assessment and cognitive training: a systematic review of applications and efficacy. FMIR Serious Games 4, 2 (2016).

[8] Lynn B. Panton and Brittany S. Loney. 2012. Exercise for Older Adults. Florida State Univerisity, College of Medicine, Department of Nutrition, Food \& Exercise Sciences.

[9] Ganit Richter, Daphne R. Raban, and Sheizaf Rafaeli. 2015. Studying Gamification: The Effect of Rewards and Incentives on Motivation. In Gamification in Education and Business, Torsten Reiners and Lincoln C. Wood (Eds.). Springer International Publishing, Cham, 21-46.

[10] Thomas Rist, Andreas Seiderer, Stephan Hammer, Marcus Mayr, and Elisabeth André. 2015. CARE: Extending a Digital Picture Frame with a Recommender Mode to Enhance Well-being of Elderly People. In PervasiveHealth '15. 112-120.

[11] Richard M. Ryan and Edward L. Deci. 2000. Intrinsic and extrinsic motivations: Classic definitions and new directions. Contemporary educational psychology 25, 1 (2000), 54-67.

[12] Hao Wang and Chuen-Tsai Sun. 2011. Game reward systems: Gaming experiences and social meanings. In DIGRA 2011.

[13] Oren Zuckerman and Ayelet Gal-Oz. 2014. Deconstructing gamification: Evaluating the effectiveness of continuous measurement, virtual rewards, and social comparison for promoting physical activity. Personal and Ubiquitous Computing 18, 7 (December 2014), 1-28.

\section{COMPETING INTERESTS}

The authors have declared that no competing interests exist. 